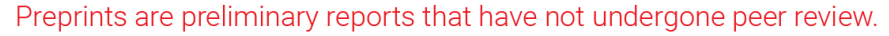 \\ They should not be considered conclusive, used to inform clinical practice, or referenced by the media as validated information. \\ CUBN Gene Mutations May Cause Focal Segmental Glomerulosclerosis (FSGS) in Children
}

\author{
Jing Yang \\ Huazhong University of Science and Technology \\ Yongli $\mathrm{Xu}$ \\ Huazhong University of Science and Technology \\ Linxia Deng \\ Huazhong University of Science and Technology

\section{Luowen Zhou} \\ Huazhong University of Science and Technology

\section{Liru Qiu} \\ Huazhong University of Science and Technology

\section{Yu Zhang} \\ Huazhong University of Science and Technology \\ Jianhua Zhou ( $\nabla$ jhzhou99@qq.com ) \\ Huazhong University of Science and Technology
}

\section{Research Article}

Keywords: CUBN gene, gene mutation, focal segmental glomerulosclerosis, proteinuria, podocyte

Posted Date: September 3rd, 2021

DOI: https://doi.org/10.21203/rs.3.rs-753923/v1

License: (c) (i) This work is licensed under a Creative Commons Attribution 4.0 International License.

Read Full License

Version of Record: A version of this preprint was published at BMC Nephrology on January 3rd, 2022. See the published version at https://doi.org/10.1186/s12882-021-02654-x. 


\section{Abstract}

Background: $C U B N$ gene mutation is extremely rare and thought to only presented as tubular proteinuria without glomerular involvement. Here, we present 3 patients with prominent proteinuria and FSGS in renal pathologies caused by novel CUBN gene mutations.

Method: Whole exome sequencing was performed in three children. CUBN gene mutations were found and then verified by sanger sequencing. Their clinical, pathological and molecular genetic characteristics were analyzed and correlated accordingly.

Results: All three children presented with prominent proteinuria but normal or slightly higher levels of urine $\beta 2$ microglobulin, without megaloblastic anemia. Renal biopsies showed segmental glomerular sclerosis, glomerular mesangial cells proliferation, effacement of foot processes, podocyte microvillation and interstitial fibrosis. There were four novel CUBN gene mutations in our study, including c.9287T $>C$ (p.L3096P), c.122+1G>A, c.7906C>T (p.R2636*), c.10233G>A (p.W3411*). Except for intron splice-site mutation, all other variants are located in highly conserved sites of CUB domain.

Conclusion: In this study, four novel pathogenic mutations of $C U B N$ gene were identified in three prominent proteinuric children. The results demonstrate that $C U B N$ gene mutations may cause pathological changes of podocytes and FSGS, which was not previously reported. Our cases extend the spectrum of renal manifestation and genotype of $C U B N$ gene mutation.

\section{Introduction}

Imerslund-Gräsbeck Syndrome (IGS) is a rare autosomal recessive disease caused by mutations of CUBN gene or $A M N$ gene $[1,2]$. The clinical features of IGS include selective intestinal vitamin B12 malabsorption, resulting in megaloblastic anemia, failure to thrive, recurrent infections, and neurological damage, with or without proteinuria, renal function is normal [3]. To date, only 48 different mutations in $C U B N$ and 32 different variants in $A M N$ have been reported in the "Human Gene Mutation Database" (HGMD) worldwide (http://www.hgmd.cf.ac.uk/ac/index.php).

CUBN gene encodes a large $460-\mathrm{kDa}$ glycosylated extracellular protein called cubilin, which is composed of 27 C-terminal CUB domains [4]. Cubilin can serve as the receptor of the intrinsic factor-vitamin B12 complex [5], and was found to express in both the renal proximal tubule [4] and podocytes [6]. There are eight $\mathrm{Ca}^{2+}$-binding epidermal growth factor repeats and a terminal region involved in trimerization of the protein in the N-terminal region [7]. CUB domains act as ligand-binding sites in the structure of cubilin, it is identified that the intrinsic factor-B12 complex can bind to the CUB domains 5-8 [8]. Cubilin has no transmembrane domain in protein structure, so it is dependent on additional factors for membrane anchoring and for endocytosis of the receptor-bound ligands [7]. As an endocytic receptor, cubilin mediates the uptake of proteins and protein-bound substances both in the intestine and in the kidneys [7]. The ligands of cubilin include intrinsic factor-B12 complex [9], albumin [10,11], vitamin carrier proteins, lipoproteins, other carriers, immune- and stress-related proteins and drugs [12]. The majority of albumin is 
absorbed through cubilin-mediated endocytosis in the proximal tubule, resulting in very low level of albumin in final urine.

In addition to Imerslund-Gräsbeck Syndrome, CUBN gene mutation has been reported in only a few cases with tubular proteinuria, and the prognosis is benign. In this paper, three $C U B N$ gene-mutated patients were presented with FSGS in renal pathologies, which was not previously reported. In addition, four novel mutations of $C U B N$ gene were found in these patients. Our cases extend the spectrum of renal manifestation and genotype of $C U B N$ gene mutation.

\section{Materials And Methods \\ 2.1 Patients}

A 6 -year- old girl and two boys aged 11 and 8 years were hospitalized due to persistent moderate proteinuria. They were regularly followed-up at the Division of Pediatric Nephrology, Tongji hospital affiliated to Tongji Medical College, Huazhong University of Science \& Technology, Wuhan, China.

\subsection{Clinical data collection, renal histopathological evaluation and follow-up study}

All three cases were routinely evaluated and regularly followed up in clinical examination, blood biochemistry, serum immunoglobulin and complement, urine albumin and $\beta 2$ microglobulin measurement, etc. Kidney biopsies were performed to evaluate the renal histopathological characteristics due to steroid resistant proteinuria. Tacrolimus was then added in these cases at dosage of $0.05-$ $0.1 \mathrm{mg} / \mathrm{kg}$ to maintain a stable trough concentration at $5-10 \mathrm{ng} / \mathrm{ml}$ for at least 3 months to observe its effect on proteinuria.

\subsection{Detection and analysis of $C U B N$ gene variant by family trio whole exome sequencing}

After the informed consent of the patients' parents, we collected blood samples from the patients and their parents. Total DNA was extracted from peripheral blood using the QIAamp DNA Blood Kit (Qiagen) according to the kit standard protocol instruction. DNA were sheared with Covaris LE220 Sonicator (Covaris) to target of 150-200bp average size. PCR amplification is performed using universal primers complementary to the adapter sequence to form a sequencing library. The sample genetic fingerprint was detected by Fluidigm Biomark using human specific SNP primers. The sequencing quality was determined by FastQC software performed on sequencing rawData. After data filtration, the clean reads were mapped to human reference genome (hg19) using Sentieon BWA software. Then, the mapped reads were detected to find SNV and InDel with Sentieon (the same algorithm with GATK) analysis, and annotated using ANNOVAR/VEP software. The pathogenic variants were screened by ClinVar, OMIM and HGMD. Functional prediction of missense mutation was determined by PolyPhen-2 and Sorting Intolerant from Tolerant (SIFT). Splice-cite prediction was determined by Human Splicing Finder (http: 
//www.umd.be/HSF/). The variants were evaluated by Clinic Sequence Analyzer (CSA) and classified according to "Standards and guidelines for the interpretation of sequence variants: a joint consensus recommendation of the American College of Medical Genetics and Genomics and the Association for Molecular Pathology" (published by ACMG, 2015). The variants were named after HGVS policy. All the suspicious pathogenic variants were validated in patient and parents using Sanger sequencing. Position of CUBN mutation sites along the cubilin protein domain were mapped using DOG 2.0(Visualization of Protein Domain Structures) [13]. Conservation of the mutation sites between different species was assessed using UCSC Genome Browser(http://genome.ucsc.edu/).

Sequences were submitted to the Swiss-Model server (https://swissmodel.expasy.org/) for modelling in automated mode, predicted effects of CUBN variants on protein structure were modelled using SwissPdbViewer [14].

\section{Results}

\subsection{Clinical, renal pathological and follow-up findings}

The clinical and renal pathological features were summarized in Table 1. All cases presented with prominent proteinuria $2+$ or over, urine $\beta 2$ microglobulin was within the normal range in 2 cases and slightly increased in 1 case. Their urine protein electrophoresis showed albumin predominantly. Their hemoglobin $(\mathrm{Hb})$ counts, serum IgG, IgA, IgM, C3, C4, and vitamin B12 levels were normal. Their renal ultrasonography showed no abnormalities. Renal biopsies showed segmental glomerular sclerosis (shown in Fig. 1A), crescents (shown in Fig. 1B, 1C), interstitial fibrosis (shown in Fig. 1D). No immunoglobulin and complement deposit were observed. Effacement of foot processes and podocyte microvillation were observed under electron microscopy (shown in Fig. 1E, 1F). Neither of their parents has proteinuria, their family diagram of the three patients shown in Fig. 2. 
Table 1

Clinical features of three patients

\begin{tabular}{|c|c|c|c|}
\hline Patient & One & Two & Three \\
\hline Age at onset(years) & 11 & 6 & 8 \\
\hline Gender & Male & Female & Male \\
\hline Edema & No & No & No \\
\hline Megaloblastic anemia & No & No & No \\
\hline proteinuria & $2+$ & $2+$ & $2+$ \\
\hline Urine albumin-creatinine & $354 \mathrm{ug} / \mathrm{mg}$ & $536 \mathrm{ug} / \mathrm{mg}$ & $462 \mathrm{ug} / \mathrm{mg}$ \\
\hline Urine $\beta 2$-microglobulin & $<0.1 \mathrm{mg} / \mathrm{L}$ & $<0.1 \mathrm{mg} / \mathrm{L}$ & $0.56 \mathrm{mg} / \mathrm{L}$ \\
\hline Urinary protein electrophoresis & $93.3 \%$ albumin & $89.3 \%$ albumin & $90 \%$ albumin \\
\hline Albumin (g/L) & 42.5 & 42.4 & 42.5 \\
\hline eGFR(ml/min.1.73m²) & 132 & 165 & 168 \\
\hline \multicolumn{4}{|l|}{ LM } \\
\hline Mesangial cell proliferation & Yes & Yes & Yes \\
\hline $\begin{array}{l}\text { Segmental sclerosis } \\
\text { (proportion) }\end{array}$ & $2 / 24$ & $1 / 40$ & $1 / 55$ \\
\hline Interstitial fibrosis & Yes & Yes & Yes \\
\hline Crescent (number) & 1 & 1 & 0 \\
\hline \multicolumn{4}{|l|}{ EM } \\
\hline Effacement of foot processes & Yes & Yes & Yes \\
\hline Podocyte microvillation & Yes & Yes & Yes \\
\hline \multicolumn{4}{|l|}{ Gene mutation } \\
\hline \multirow[t]{2}{*}{ Allele 1} & c. $.9287 \mathrm{~T}>\mathrm{C}$, & c. $9287 \mathrm{~T}>\mathrm{C}$, & c. $10233 \mathrm{G}>\mathrm{A}$, \\
\hline & p. L3096P (Exon59) & $\begin{array}{l}\text { p. } \\
\text { L3096P(Exon59) }\end{array}$ & $\begin{array}{l}\text { p.W3411* } \\
\text { (Exon64) }\end{array}$ \\
\hline \multirow[t]{2}{*}{ Allele 2} & \multirow{2}{*}{$\begin{array}{l}\text { c. } 122+1 G> \\
A(\text { Intron } 1)\end{array}$} & c. $7906 \mathrm{C}>\mathrm{T}$, & c. $10233 \mathrm{G}>\mathrm{A}$ \\
\hline & & $\begin{array}{l}\text { p.R2636* } \\
\text { (Exon51) }\end{array}$ & $\begin{array}{l}\text { p.W3411* } \\
\text { (Exon64) }\end{array}$ \\
\hline
\end{tabular}

\subsection{Identification of CUBN gene Mutation}


CUBN gene mutations were identified by family trio WES (whole exome sequencing) of three children and their parents. Patient 1 carried compound heterozygous CUBN gene mutation c.9287T > C (p.L3096P) and c. $122+1 \mathrm{G}>\mathrm{A}$, which came from his father and mother respectively (shown in Fig. 3A). The former was a novel missense mutation of $C U B N$ gene, not seen in ExAC database, with a frequency of 0.0001 in 1000 genomes database. Poly-phen2, SIFT, Mutation Taster software revealed the scores were 0.813 (possibly damaging), 0.003 (damaging), 1.000(disease causing) respectively. This missense mutation was regarded as possibly pathogenic (PM2, PM3, PP3, and PP4), the other splice-site mutation was predicted as "most probably affecting splicing" by Human Splicing Finder, and regarded as pathogenic (PVS1, PM2, and PP4) according to ACMG criteria.

Patient 2 carried compound heterozygous CUBN gene mutation c.9287T > C (p.L3096P) and c.7906C > T (p.R2636*), which came from his father and mother respectively (shown in Fig. 3B). The former was the same missense mutation as in patient 1 . The other was a nonsense mutation leading to truncation of the protein. The frequency of this mutation was 0.00007 in ExAC and 0.00019 in 1000 genomes database. Mutation Taster, CADD and DANN software showed the mutation scores were 1.000 (disease causing), 55(damaging), 0.998(damaging). The nonsense mutation was regarded as pathogenic (PVS1, PM2, and PP4) according to ACMG criteria.

Patient 3 carried homozygous CUBN gene mutation c.10233G > A (p. W3411*) (shown in Fig. 3C). It was a nonsense mutation leading to cubulin truncation, not seen in both ExAC and 1000 genomes databases. Mutation Taster, CADD, DANN software revealed the mutation scores were 1.000(disease causing), 51 (damaging), 0.993(damaging). This novel mutation was regarded as pathogenic (PVS1, PM2, and PP4) according to ACMG criteria. Except for the splicing mutation in patient 1, all other mutations were located at CUB domains 19, 23, 27 of cubilin (shown in Fig. 3D). UCSC Genome Browser alignment indicated that L3096, R2636 and W3411 in CUBN gene was highly conserved among different species (shown in Fig. 3E).

Since two patients shared a same novel missense mutation (c.9287T > C, p.L3096P), we built 3Dstructure model of the mutant cubilin protein based on the solved CUBN 3D-structure by Swiss-Model (shown in Fig. 4A), as they share high sequence identity (6fzv, 36.25\%). The 3D-structure model and surface model between wild-type cubilin protein and mutant cubilin protein were showed in Fig. 4B. Calculated the hydrogen bond between the wild type and the mutant, and found that the hydrogen bond has changed.

\section{Discussion}

Previous studies have demonstrated that CUBN mutations can cause IGS, the common clinical features of IGS include megaloblastic anemia and secondary neurological symptoms, with or without proteinuria. Isolated proteinuria caused by CUBN mutation is rare, only a few cases have been reported in the world so far. Boger et.al [15] identified a missense mutation (I2984V) in the CUBN gene which is associated with albuminuria in both the general population and in individuals with diabetes. In 2011, Ovunc et.al [3] 
revealed that cubilin mutation as a single-gene cause of proteinuria by exome sequencing. As for the underlying mechanism of proteinuria, it's speculated that mutated CUBN might affect protein reabsorption in the proximal tubules. As a ligand of cubilin, albumin binds to it at the proximal tubule, so there is almost no albumin in the normal urine. Therefore, when cubilin is defective, the uptake of albumin by the proximal tubule cells will be significantly reduced, resulting in proteinuria. Amsellem et.al [16] suggested that selective daily albumin excretion was increased approximately six-folds in cubilindeficient mice. The cause of albuminuria is still controversial. Our cases demonstrate the possibility that CUBN geme mutations may pathologically present with pathological changes of podocytes and FSGS. The albuminuria was probably not only originated from renal proximal tubule loss but also from podocyte disfunction. As a podocyte-targeting immunosuppressive agent, tacrolimus could significantly reduce and even normalize the levels of albuminemia, which further supports the important role of podocyte in the pathogenesis of proteinuria in $C U B N$ mutated patients.

Due to the scarcity of cases with CUBN gene mutation, kidney biopsy has been performed in only a few patients so far. In addition to isolated proteinuria, the renal pathology of our patients showed abnormal changes of podocytes. Until now, focal segmental glomerulosclerosis has not been reported in patients with CUBN gene mutation [17]. In 2012, Prabakaran et.al [6] found cubilin expression in rat podocytes and human podocytes. In rats, cubilin was expressed in the plasma membrane, vesicles and multivesicular bodies. Gianesello et.al [18] proved that cubilin mediates albumin endocytosis in human podocytes, CUBN gene mutation may lead to the dysfunction of cubilin, thus affect albumin endocytosis. The development of proteinuria is usually related to podocyte damage, such as podocyte foot process effacement and cell loss [19]. Compared to megalin, cubilin is thought to have higher binding affinity for albumin [20]. Megalin can function as a sensor of albumin to determine the effect on cell survival. No or only a small amount of albumin binding to megalin can inhibit podocyte apoptosis, however, a large quantity of albumin binding to megalin can promote cell apoptosis [6]. It's thought that the CUBN mutation leads to reduction of binding capacity of cubilin to albumin, which makes free albumin available to bind with megalin. Consequently, it promotes podocyte apoptosis via the pl-3K/PKB pathway, leading to a decreased number of podocytes. As a kind of terminal differentiated cell, podocyte can't proliferate to compensate the loss of podocyte, thereafter glomerular basement membrane become naked, followed by FSGS. Bedin et.al [17] reported the renal pathologies of the patients with CUBN mutations, most of them were minimal change disease or no lesions. But there were two patients whose lesions tended to be in the early stage of FSGS [17], which is consistent with our finding. Interestingly, those pathologically manifested with FSGS were unanimously have at least a relatively serious mutation in one allele, such as nonsense mutation, insertion, deletion or mutation in splice sites which may lead to frameshift or protein truncation. Since renal biopsy was rarely performed in patients with $C U B N$ gene mutation, even less for electron microscopy (EM) examination. Therefore the pathological changes of podocytes were not noted on EM in the past. The obvious podocyte abnormalities in our cases provide evidence for its involvement in the pathogenesis of albuminuria in CUBN gene mutated patients.

Bedin et.al [17] firstly reported that biallelic CUBN mutations could cause isolated proteinuria, and proteinuria-associated CUBN mutations were localized to C-terminal CUBN domains. Our patients showed 
obvious proteinuria, but without megaloblastic anemia. Except for one intron splice-site mutation, all other mutations are located in highly conserved sites at the C-terminal, CUB19, CUB23 and CUB27 domain respectively, which is consistent with the results of Bedin. All our cases had at least one relatively serious mutation and severe pathological changes accordingly.

In this study, four novel pathogenic mutations of $C U B N$ gene were identified in three prominent proteinuric children. The results demonstrate that, in addition to previous phenotypes, the novel serious CUBN gene mutations may cause pathological changes of podocytes and FSGS, which was not previously reported. Our cases extend the spectrum of renal manifestation and genotype of CUBN gene mutation.

\section{Abbreviations}

FSGS: focal segmental glomerulosclerosis; CSA: Clinic Sequence Analyzer; ACMG: American College of Medical Genetics; IGS: Imerslund-Gräsbeck Syndrome; EM: electron microscopy.

\section{Declarations}

\section{Ethics approval and consent to participate}

This study was approved by The Human Ethics Committees of Tongji Hospital, Tongji Medical College, Huazhong University of Science and Technology.

\section{Consent for publication}

We obtained written informed consent for publication of the article, including any identifiable data and images from the parents of the three patients.

\section{Availability of data and materials}

The data that support the finding of this study are available from the corresponding author upon reasonable request.

\section{Competing interests}

The authors declare that they have no competing interests.

\section{Funding}

This work was supported by a grant (No.81873596) from the National Natural Science Foundation of China to Dr. Jianhua Zhou. 


\section{Author Contributions}

All authors contributed to the intellectual content of this manuscript and approved the final manuscript as submitted. JY collected data and drafted the manuscript with the help of LD, LZ and JZ, LQ and YZ performed pathological analysis, JZ interpreted the data and revised the article for important intellectual content. All authors have critically read and approved the manuscript.

\section{Acknowledgement}

We thank all patients and their families for the participation in this study.

\section{References}

1. Aminoff M, Carter JE, Chadwick RB, Johnson C, Grasbeck R, Abdelaal MA, et al. Mutations in CUBN, encoding the intrinsic factor-vitamin B12 receptor, cubilin, cause hereditary megaloblastic anaemia 1. Nat Genet 1999; 21: 309-13.

2. Tanner SM, Aminoff M, Wright FA, Liyanarachchi S, Kuronen M, Saarinen A, et al. Amnionless, essential for mouse gastrulation, is mutated in recessive hereditary megaloblastic anemia. Nat Genet 2003; 33: 426-9.

3. Ovunc B, Otto EA, Vega-Warner V, Saisawat P, Ashraf S, Ramaswami G, et al. Exome sequencing reveals cubilin mutation as a single-gene cause of proteinuria. J Am Soc Nephrol 2011; 22: 1815-20.

4. Nielsen R, Christensen El, Birn H. Megalin and cubilin in proximal tubule protein reabsorption: from experimental models to human disease. Kidney International 2016; 89: 58-67

5. Seetharam B, Christensen El, Moestrup SK, et al. Identification of rat yolk sac target protein of teratogenic antibodies, gp280, as intrinsic factor-cobalamin receptor. J Clin Invest. 1997; 99: 23172322

6. Prabakaran T, Christensen El, Nielsen R, et al. Cubilin is expressed in rat and human glomerular podocytes. Nephrol Dial Transplant2012; 27: 3156-3159

7. Christensen El, Nielsen R, Birn H. From bowel to kidneys: the role of cubilin in physiology and disease. Nephrol Dial Transplant2013; 1-8

8. Andersen CB, Madsen M, Storm T et al. Structural basis for receptor recognition of vitamin-B(12)intrinsic factor complexes. Nature 2010; 464: 445-448

9. Christensen El, V erroust PJ, Nielsen R. Receptor-mediated endocytosis in renal proximal tubule. Pflugers Arch 2009; 458: 1039-1048

10. Birn $\mathrm{H}$, Fyfe JC, Jacobsen $\mathrm{C}$ et al. Cubilin is an albumin binding protein important for renal tubular albumin reabsorption. J Clin Invest 2000; 105: 1353-1361

11. Zhai $X Y$, Nielsen R, Birn $\mathrm{H}$ et al. Cubilin- and megalin-mediated uptake of albumin in cultured proximal tubule cells of opossum kidney. Kidney Int 2000; 58: 1523-1533 
12. Nielsen R, Christensen El. Proteinuria and events beyond the slit. Pediatr Nephrol 2010; 25: 813-822

13. Jian Ren, Longping Wen, Xinjiao Gao, Changjiang Jin, Yu Xue and Xuebiao Yao. DOG 1.0: Illustrator of Protein Domain Structure. Cell Research 2009; 19: 271-273

14. Guex N, Peitsch MC. SWISS-MODEL and the Swiss-PdbViewer: an environment for comparative protein modeling. Electrophoresis 1997; 18: 2714-23

15. Boger $\mathrm{CA}$, Chen MH, Tin A, Olden M, Kottgen A, de Boer IH, Fuchsberger C, O'Seaghdha CM, Pattaro C, Teumer A, et al. CUBN is a gene locus for albuminuria. J Am Soc Nephrol 2011; 22: 555-70

16. Amsellem S, Gburek J, Hamard G, Nielsen R, Willnow TE, Devuyst O, Nexo E, Verroust PJ, Christensen $\mathrm{El}$, and Kozyraki R. Cubilin Is Essential for Albumin Reabsorption in the Renal Proximal Tubule. J Am Soc Nephrol 2010; 21: 1859-1867

17. Bedin M, Boyer O, Servais A, Li Y, Villoing-Gaude L, Tete MJ, Cambier A, Hogan J, Baudouin V, Krid S, Antignac $\mathrm{C}$, Simons $\mathrm{M}$, et al. Human C-terminal CUBN variants associate with chronic proteinuria and normal renal function. J Clin Invest 2020; 130: 335-344

18. Gianesello L, Priante G, Ceol M, Radu CM, Saleem MA, Simioni P, Terrin L, Anglani F, and Prete DD. Albumin uptake in human podocytes: a possible role for the cubilin-amnionless (CUBAM) complex. Sci Rep 2017; 7: 13705

19. Ying Liu, Shan Li, Weiwei Rong, Caihong Zeng, Xiaodong Zhu, Qilin Chen, Limin Li, Zhi-Hong Liu, Ke Zen. Podocyte-Released migrasomes in urine serve as an indicator for early podocyte injury. Kidney Dis 2020; 6:422-433

20. Christensen El, Birn H, Storm T, Weyer K and Nielsen R. Endocytic receptors in the renal proximal tubule. Physiology (Bethesda) 2012; 27:223-236

\section{Figures}




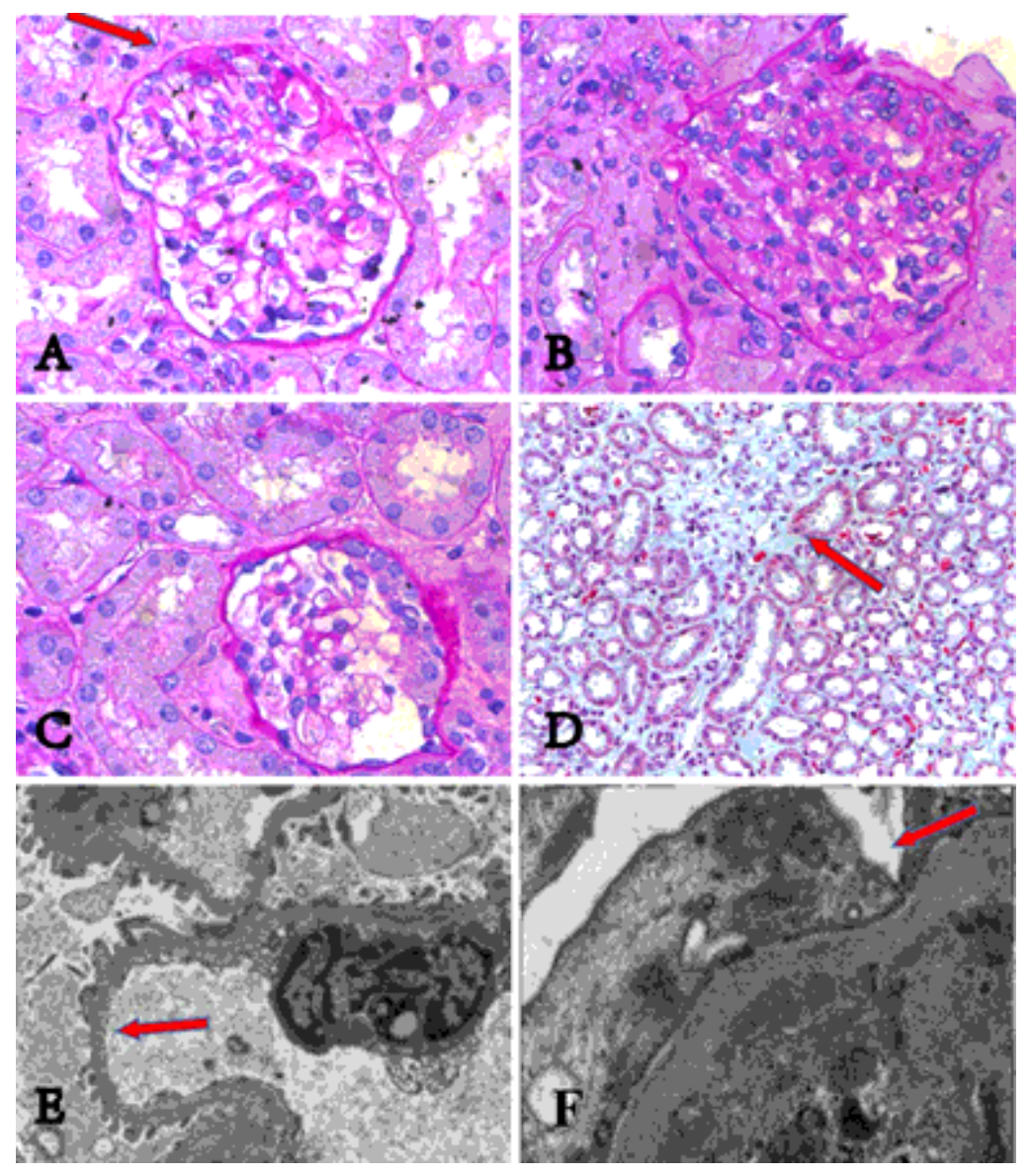

\section{Figure 1}

Pathological changes in CUBN gene mutated patients (A) segmental glomerular sclerosis (pointed by red arrow). (B and C) Crescents in patients. (D) Interstitial fibrosis (pointed by red arrow). (E) Podocyte microvillation (pointed by red arrow). (F) Effacement of foot processes (pointed by red arrow).

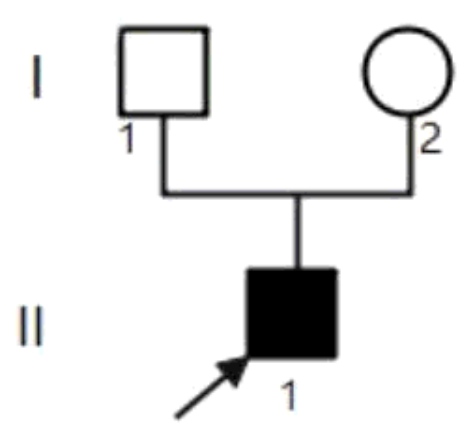

A

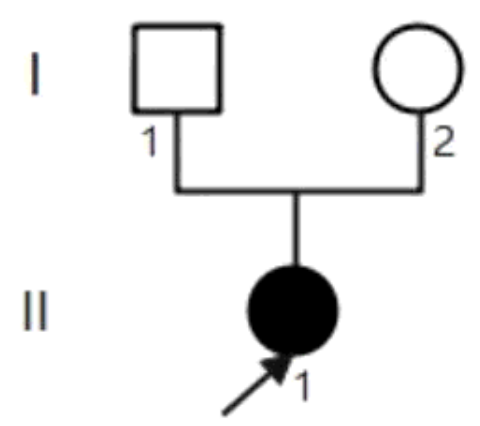

B

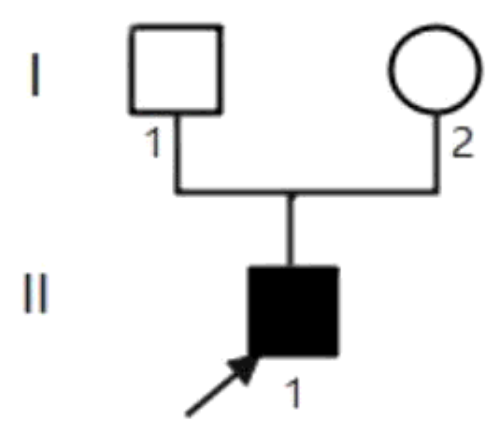

C

\section{Figure 2}

Family diagram of the three patients. A, B, C indicate patient 1, 2, 3 respectively. 


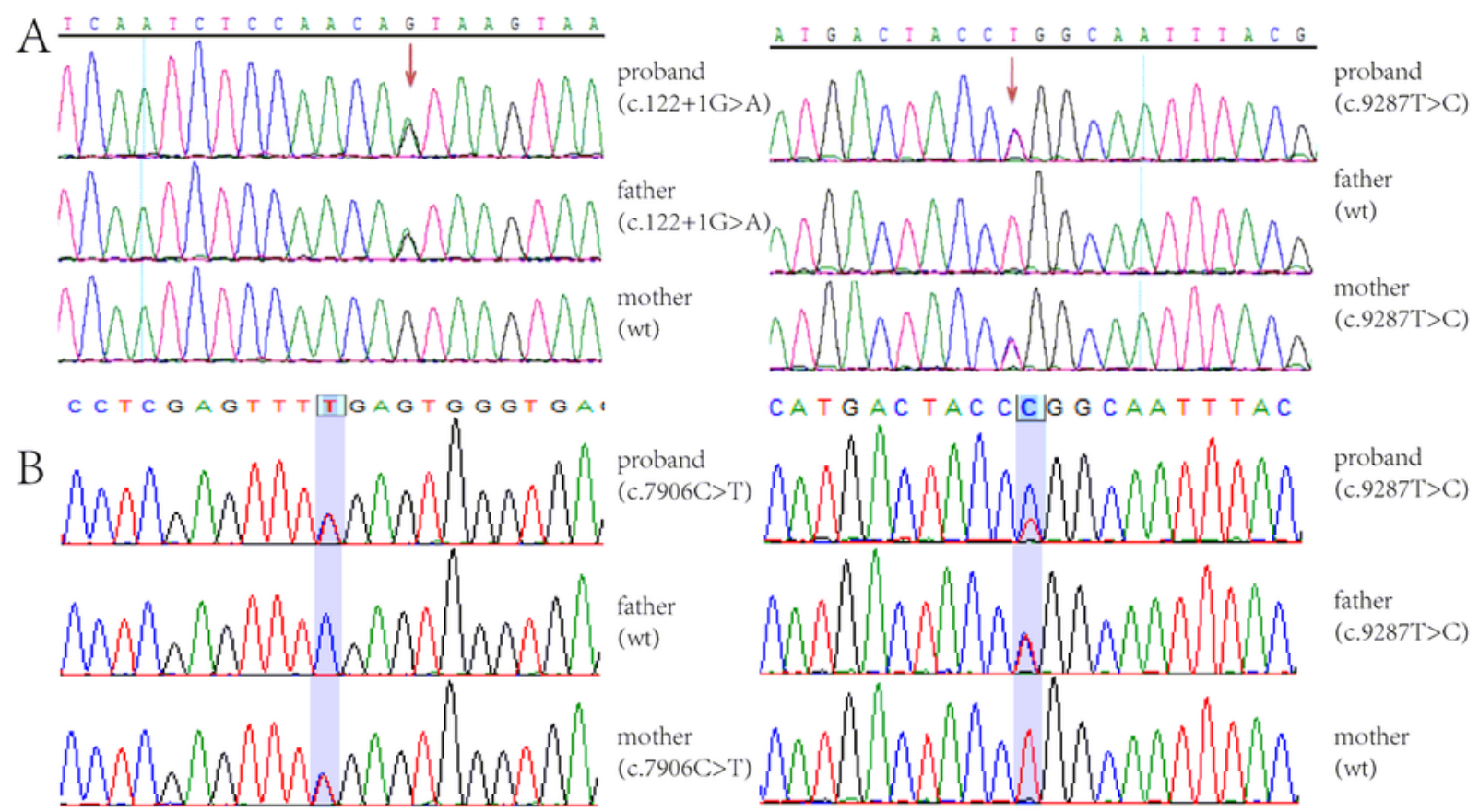

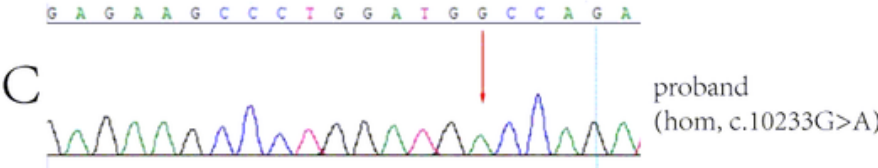

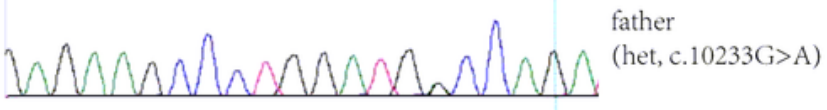

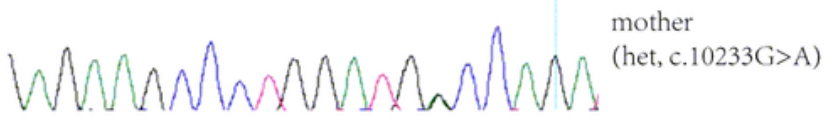

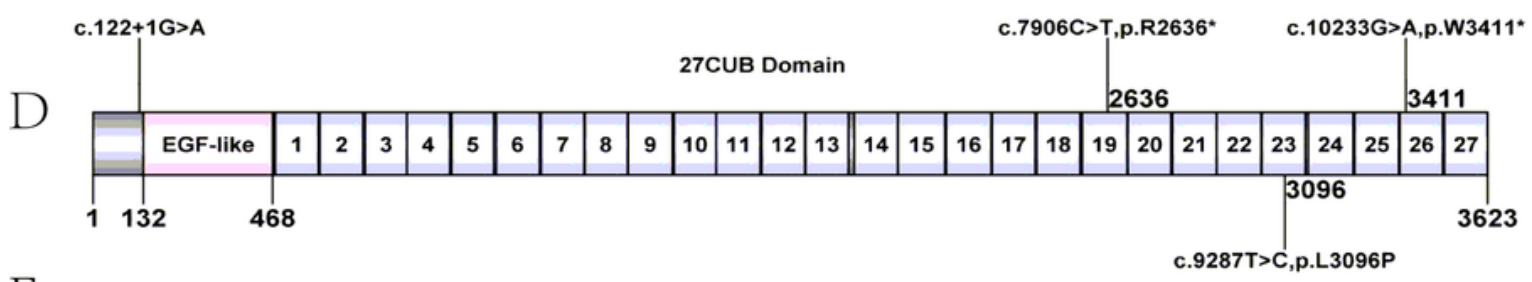

E

\begin{tabular}{|c|c|c|c|c|}
\hline & & 13411 & L3096 & R2636 \\
\hline Human & $D N D Y N D P$ & W G P S R L N & $A G D Y I A L Y D H S C S T$ & VRFELVDFQCDQHS \\
\hline hesus & $G N N Y N D P$ & W G P S R L N & $A G D Y I A L Y D H S C S T$ & VRFELVDFQCDQHS \\
\hline ouse & $N$ N A Y G Q P & W G P S K L N & $P G D F V E L Y D H S C L N$ & VRFELVDFTCDOHS \\
\hline $\mathrm{gg}$ & N N D Y N D P & W G P S K L N & $S G D Y I A L Y D Q S C F T$ & VRFELVDFQCDQHS \\
\hline I ephant & $D N N Y S D P$ & W G P S K L N & $S G D Y I A L Y D Q S C F A$ & VRFELVDFQCDQHS \\
\hline
\end{tabular}

Figure 3

Confirmation of conservation analysis of CUBN gene mutations in three families. (A) Compound heterozygous mutation of CUBN gene in patient 1 , his patients carry heterozygous CUBN gene mutation of c. $122+1 \mathrm{G}>\mathrm{A}$ and c.9287T $>C$, respectively. (B) Compound heterozygous mutation of CUBN gene in patient 2, his patients carry heterozygous CUBN gene mutation of c.7906C $>$ T and c.9287T >C, respectively. (C) Sanger sequencing shows c.10233G>A mutation in patient 3 and his patients. (D) 
Position of the CUBN variants along the cubilin protein. (E) Conservation of L3096, R2636 and W3411 (blue rectangle showed) in CUBN gene. Wt: wild type; hom: homozygous; het: heterozygous

\section{A}

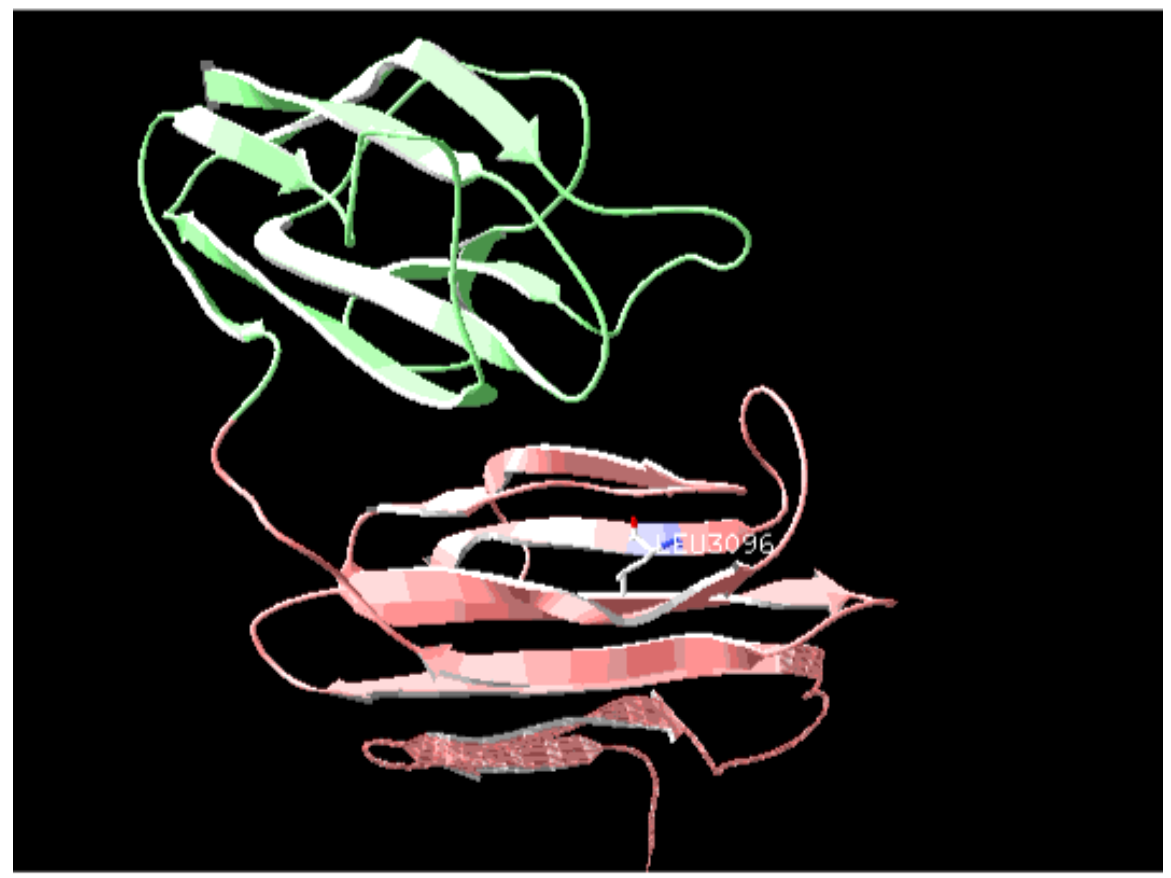

B
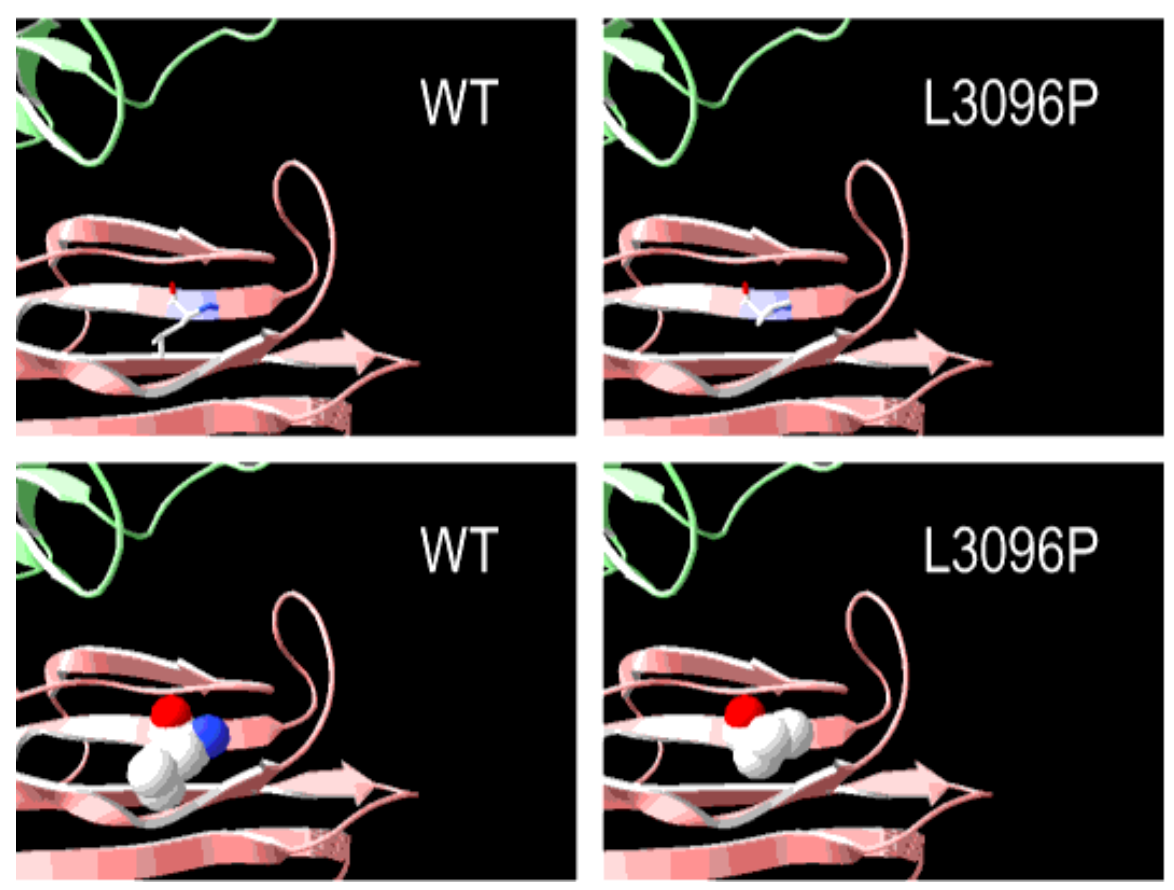

Figure 4

Protein modelling of CUBN variants. (A) CUBN are shown in ribbon format, colored green, pink, and purple, the purple indicates CUBN residue 3096. (B) The 3D-structure model and surface model between wild-type cubilin protein and variant cubilin protein. 